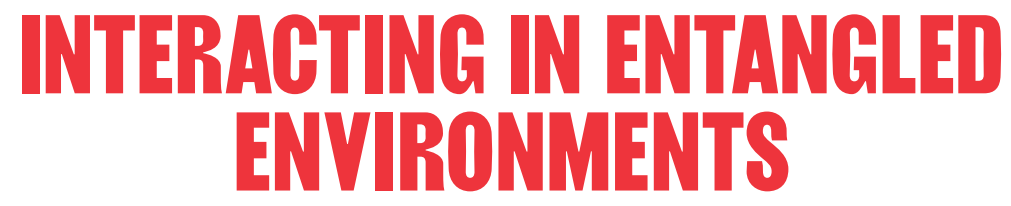


FIG. 1

Immersed in the sulphuric environment of the Solfatara Crater, Pozzuoli, Italy, 2017 (Photo: Roman Kirschner).

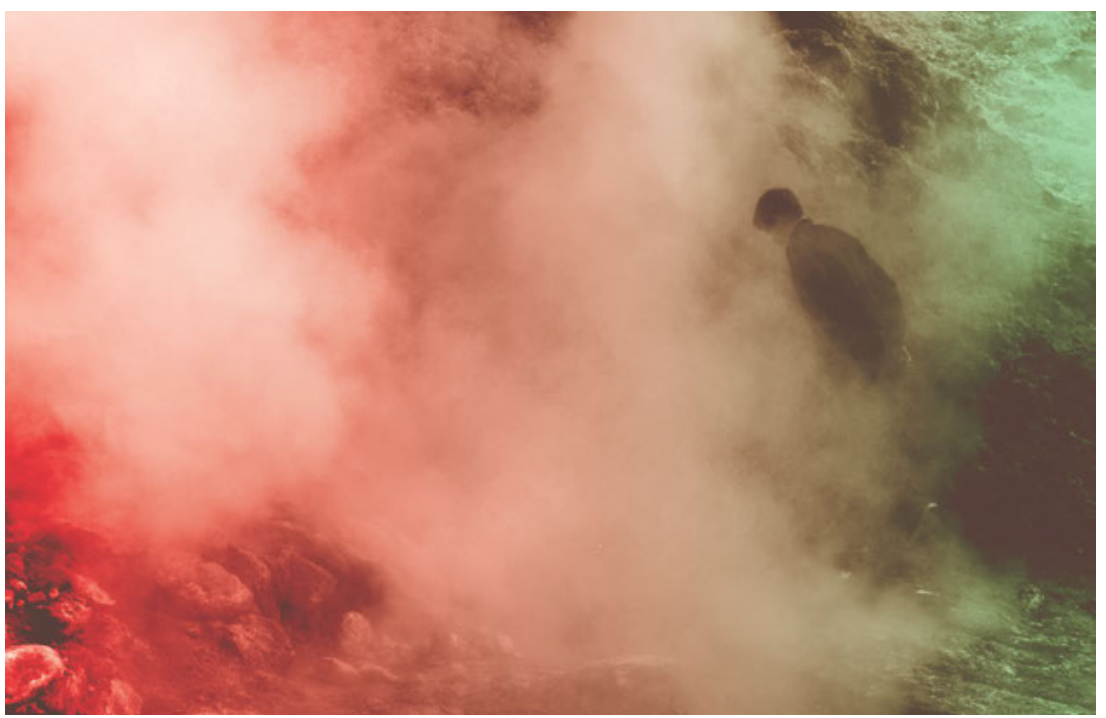


We live in a material world full of dynamics. Let's pay tribute to this fact. Let's embrace its flows of matter and energy, its transformations and its variety. Let's collaborate with its complexity and entanglements!

There is a fundamental shortcoming in mainstream design practices that "aspire toward a logic of form that reduces our ability to perceive the depth and scope of our material involvement with the world around us. [...] The effect is to trap humanity within a vicious circle of increasing environmental alienation." vironment and our lack of respect for its offerings and limitations are coupled with another startling insight from environmental ecology. Historical analysis shows that technological development in societies leads to an ever-increasing per capita consumption of energy and material resources. 然 We are literally devouring our planet. To give just one example: industrial agriculture requires such massive amounts of fossil fuels that it turns into an energetic loss. In France, the United States and Denmark, one calorie of energy is transformed into 0.7 calories of nutrition. in the efficiency of technical processes doesn't seem to be able to decouple the growing societal metabolism from economic development. Could a more ecological, ecosystemic approach offer a way out of this vicious techno-political circle?

\section{THE AGE OF ENTANGLEMENT}

Our societies are no longer organised on the basis of deep ancestral connections with our closest environment. We have new ways of collecting data about our planet and of expanding our living environments and our radius of action. Machine-based information systems are organised in massive networks and are growing in importance. Some

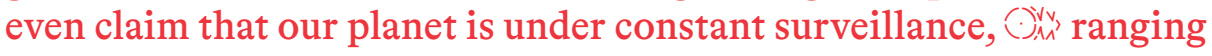
from small mobile devices roaming the surface to the many satellites in orbit looking down on us. The interconnectedness of these machines, and the increasing complexity faced by our societies, have led some researchers to believe that we have entered a new "age of entanglement." $\equiv>>$ We believe that this notion of entanglement should not be based solely on technological aspects and solutions, but also on the 
impact that our daily actions have on the complex web of life, whether that impact be immediate, mediated, deferred, collective or individual. When we consider that our planet is an almost closed system, we should always keep planetary ecologies in view. The "age of entanglement" must take into consideration those ways in which ecosystems and their components are entangled.

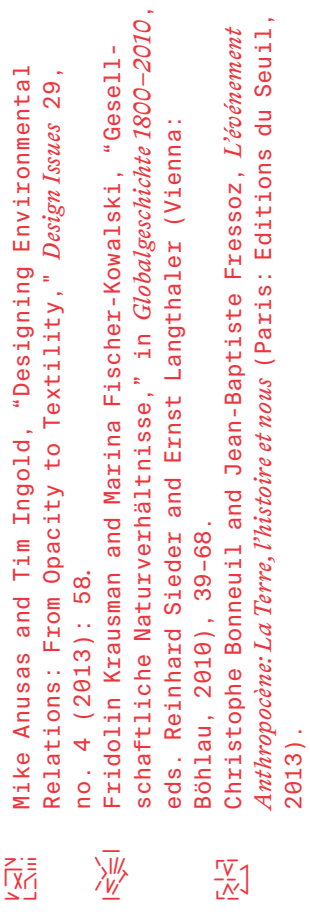

As we discover new complexities and dynamics in our living environments, it is not sufficient for us to explore the increasing complexity of human activity in interplay with complicated tools. We also need to consider how these activities are embedded in the landscapes on which they depend, how they exchange energy and materials across scales and how these flows influence other participants in shared ecosystems, both local and global. More than ever, we must not forget that our supposedly local, rather abstract actions have an impact on the environment. For example, conducting a simple Internet search generates an unexpectedly high $\mathrm{CO}_{2}$ output through the use of server power and network infrastructure, and this contributes to the immense energy consumption of the IT sector. Yet our concern with such small actions is not about multiplying their harmful consequences on a global scale and inducing guilt, but rather about questioning their inherent values and how these values guide our habits.

\section{PERMEATION AND FLOW}

Let us recall the ongoing discussions in biology concerning the basic relationship between organisms and their environments. This relationship is one of mutual entanglement. The evolution of an organism cannot be understood in isolation from its surroundings, while its sur-

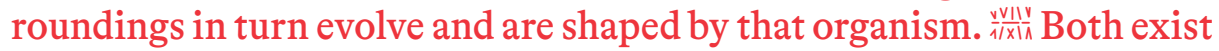
in a state of mutual co-creation. To a certain extent, one can even speak of permeation. For example, the myriads of microorganisms inhabiting the human body are for the most part not any sign of infection, but engage in an essential symbiosis by performing basic life functions. In the case of human digestion, this helps feed both humans and the microorganisms. And these microorganisms also connect us to the 
FIG. 2

Conceptual Speed Dating of Interaction Design students standing in the sea and discussing how to work with the environment. While, every eight minutes, one side moves one place further deeper into the water, the other side stays static and slowly attracts the attention of small marine lifeforms. (Photo: Karmen Franinović, 2018).

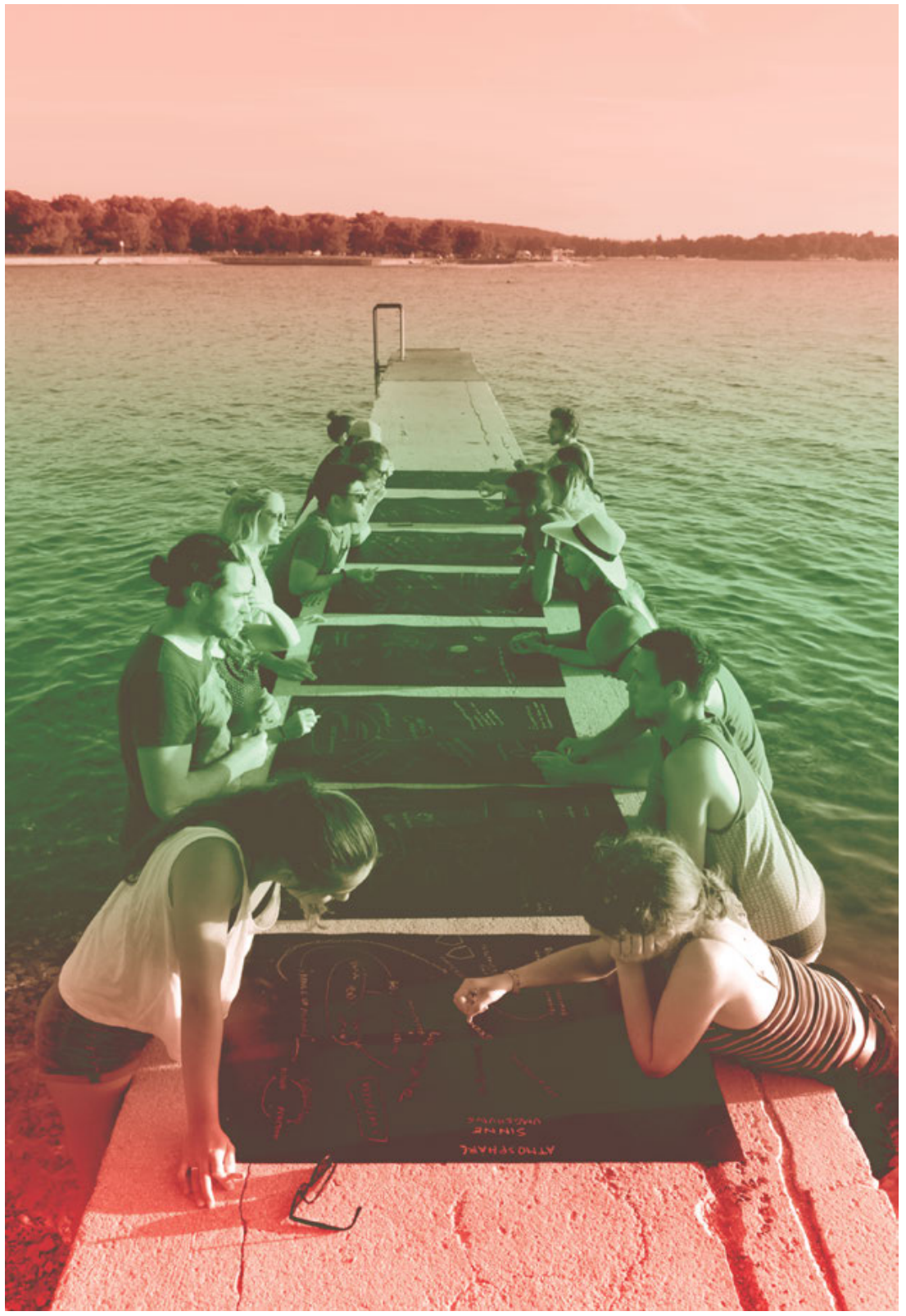


remote Waimea valley on the Hawaiian island of Oahu. Researchers discovered "that each organism's microbiome is a subset of what exists in the broader environment and in organisms lower on the food web." This offers a clear clue that the microbiome is not only seeded from the environment, but also demonstrates "how connected our world is, all

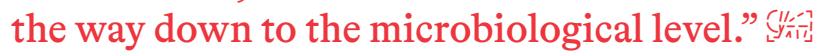

What links the above examples together is the fact

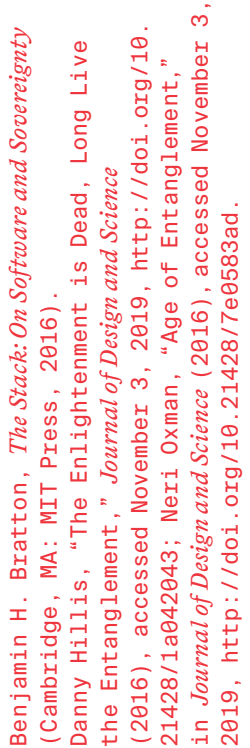
that nothing is isolated. Nothing floats in an abstract empty space, as some traditions of thought might suggest. In the first and most basic of his four "laws of ecology," the ecologist Barry Commoner wrote: "Everything is connected to everything else." (伩依 However, the notion of "omniconnectedness" needs to be refined if it is not to be abused. Donna Haraway relativises the closeness of relationships by stating that "Nothing is connected to everything, everything is connected to something!" tions can be indirect and mediated in manifold ways. They can also be different in nature: causal, functional, instrumental, incidental, periodic, parasitic or symbiotic, and so on. Our planet Earth and its atmosphere are anything but a void. Everything on our planet is embedded in flows of matter and energy. It is a dynamic environment providing an indescribable sensory and creative richness.

How can we become open to this? What can practitioners in the field of art and design do, if we assume that everything is connected to everything else and that current technological solutions are perpetuating the environmental exploitation described above? In this entangled world, do concepts of "user," "audience" or "viewer" still hold at all? How can we move away from practices that reduce our material engagement with our surroundings towards practices that foster a sense of connectedness, of caring for and enjoying the world? As a working hypothesis, we propose two starting points: attunement and leveraging.

PROPOSITION 1: ATTUNE!

Attunement is a process of opening up to the dynamics of our surroundings, becoming aware of an ecology or an environment in all its complexity, scales and depths. Designers have applied attunement almost exclusively to humans (e.g. empathic 
methods), taking living environments as a more-orless stable context within which human action takes place. However, a world of non-human organisms and phenomena, such as soil microbiomes or volcanoes, is full of activities that are not directly accessible to practitioners in art and design. An initial challenge is thus to access the dynamics of ecologies without limiting our focus to human activities and artefacts.

One way is to shift our attention and apply empathic methods to natural entities other than the human. These, however, could easily be anthropomorphised if we follow familiar methods. If one sits in the Solfatara crater knowing its stories of eruptions, listening to the bubbling, smelling the sulphur and feeling the heat, this spontaneously generates strong human sensations that might easily be transferred to the volcano itself (e.g. fear or anger). We must therefore find new ways of attuning; fields such as the arts and anthropology have been doing this, for example, by deploying soundwalks and other awareness techniques, pology beyond the human."

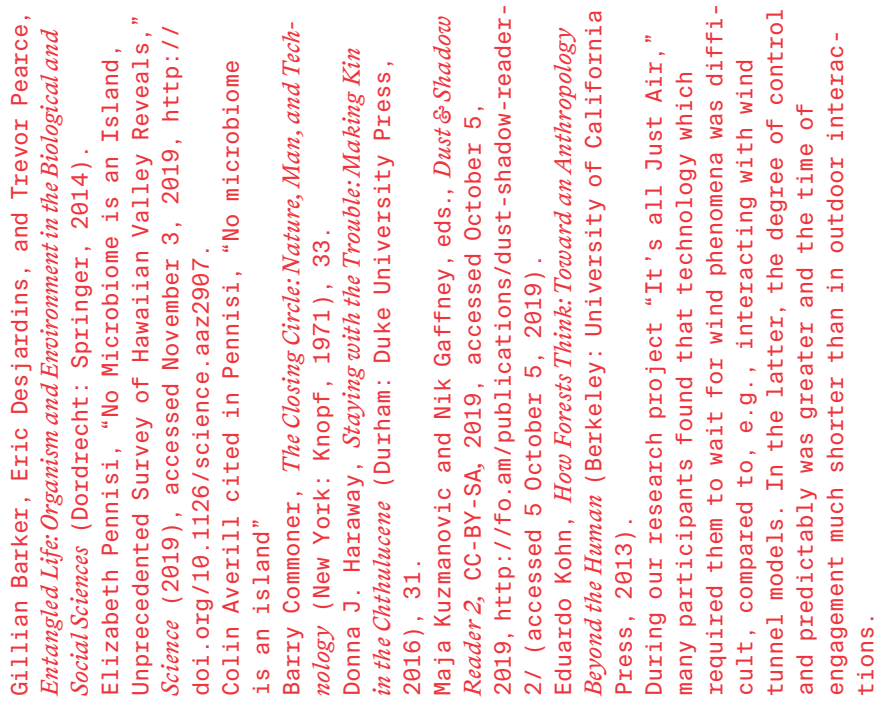

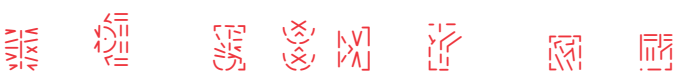

The most important aspect of attunement is to take time to be in an environment and to sense things, organisms and phenomena. This approach can be extended from field work to ideation, and further 
to interventions in existing ecologies or the creation of technologies that go far beyond gamified, user-friendly interactions. Technologies can foster patience and a connection with our surroundings (think of a telescope). But at present, our expectation of technology is that it should provide hard facts, rather than engaging in long-term physical interactions with our surroundings. quire an attitude of patience and care if we wish to understand and work with dynamic flow ecologies. A further challenge is thus to develop interventions into ecosystems that allow them to blossom, but not solely according to human needs.

\section{PROPOSITION 2: LEVERAGE!}

Our approach to intervening and collaborating with ecosystems draws on our research into materials as active partners in a conversational paradigm. ¿Cill $_{=}$We propose "leveraging" as a way of bringing the insights of attunement back into the field of making, in a way that goes beyond focusing on isolated objects and is instead geared towards dynamic ecosystems. Leveraging points are places in a complex system where "a small shift in one thing can produce big changes in everything." 구르리 The principle of leveraging was devised by Donella Meadows, one of the co-authors of the ground-breaking report of the Club of Rome. from general systems theory and has been extensively used in business and finance. Leveraging only recently found its way into sustainability

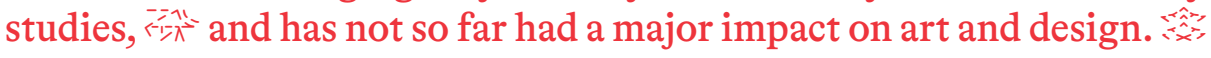

In the context of making, leveraging represents an efficient, considerate way of influencing working systems. It seems especially suitable for influencing and shaping flow ecologies. Leverage points can be found in the physical layers of a system (e.g. flows, stocks, delays, feedback, or structure in general), but also at higher levels (e.g. access to information, rules, intentions, etc.). These are points of power. Nevertheless, rather than taking control of systems, we recommend a more tempered approach: one of subtle, non-disruptive interventions. Furthermore, leverage points can also be used to amplify actions, to illustrate influences, or to show interconnections and linkages. 


\section{CONCLUSION}

Our proposals are grounded in existing entanglements as a starting point for creative practice. An increased attention to one's surroundings, an awareness of ongoing processes, and a responsiveness and a readiness to adapt on the part of researchers are key to understanding complexity beyond the numbers and models that have so often failed us, as Meadows shows. Although not quantitative, our methods require rigour and iteration as well as openness and care towards given situations. We cannot treat existing environments only as physical resources or as abstract formal contexts for the benefit of our practices, to be accessed through mediated images, maps and models. We must learn how to negotiate the complexities and unpredictabilities of entangled, spatiotemporal, material flows. We must attune and leverage!

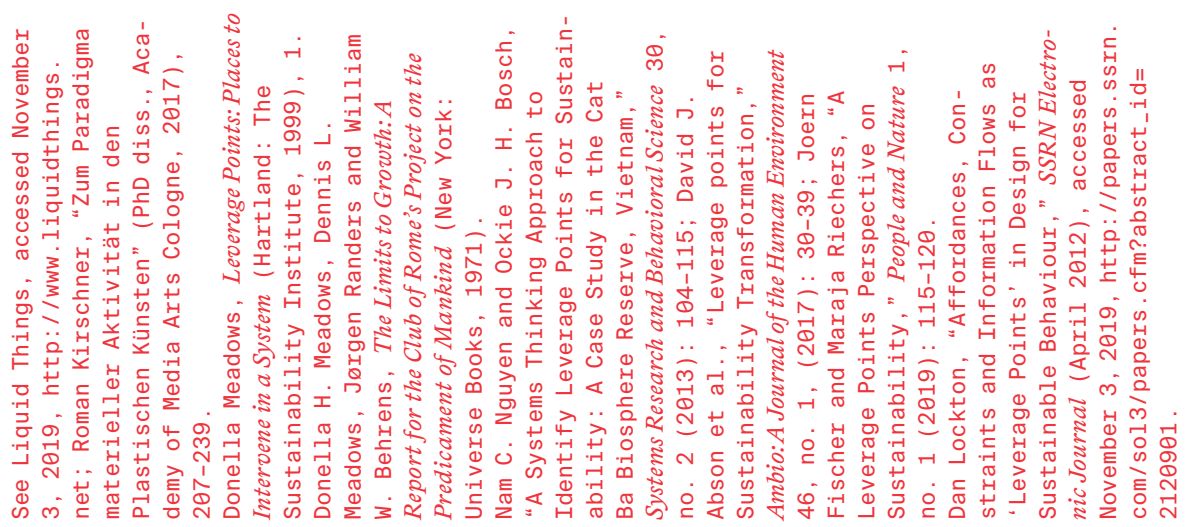





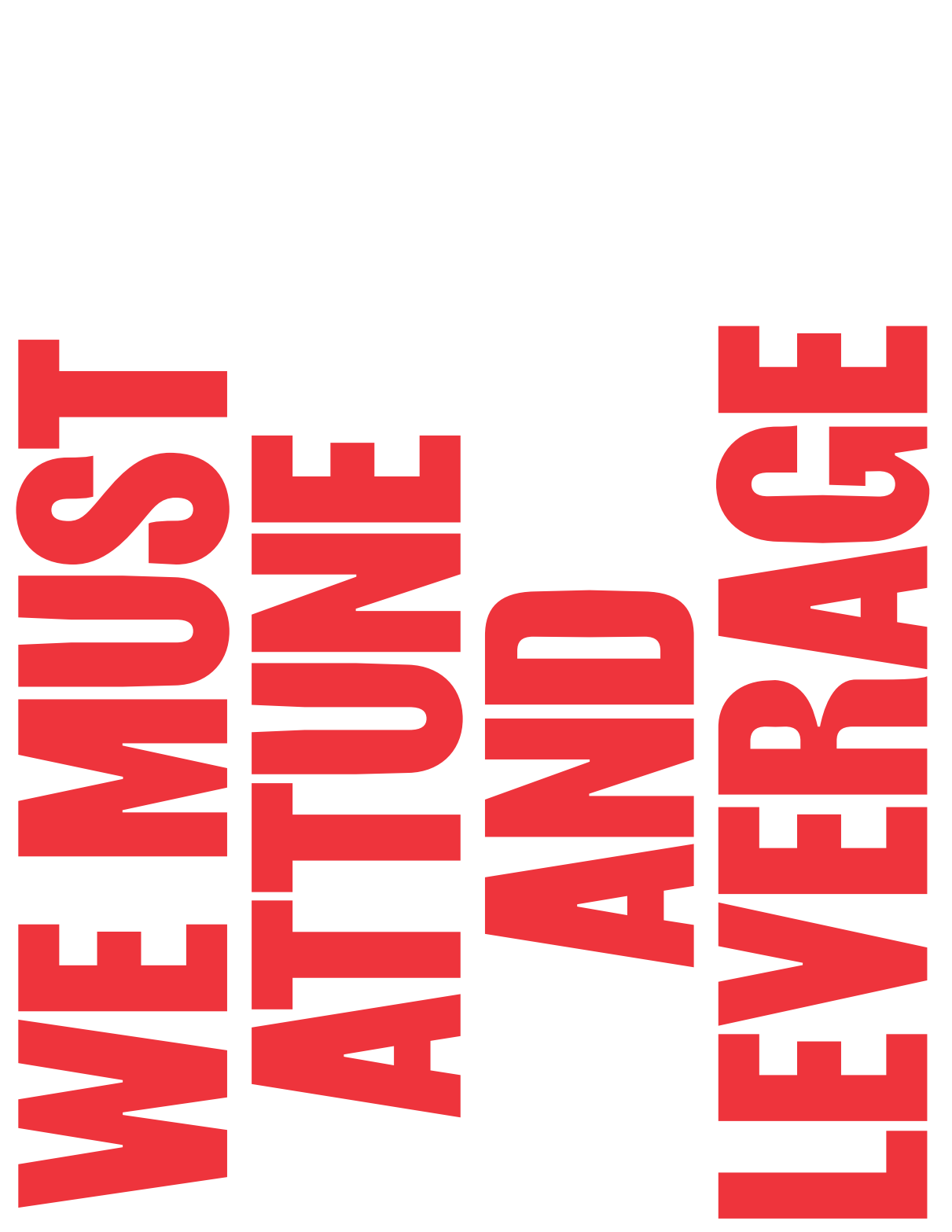


